\title{
DESEMPENHO DE FRANGOS DE CORTE CRIADOS EM DIFERENTES DENSIDADES POPULACIONAIS, NO INVERNO, NO SUL DO BRASIL
}

\author{
PERFORMANCE OF BROILERS REARED IN DIFFERENT POPULATION \\ DENSITY, IN WINTER, IN SOUTH BRAZIL
}

\section{Antônio Carlos Mortari ${ }^{1}$ Alexandre Pires Rosa ${ }^{2}$ Irineo Zanella $^{2}$ Carlos Beretta Neto $^{3}$ Paulo Roberto Visentini ${ }^{4}$ Lourdes B. Padilha Brites ${ }^{5}$}

RESUMO

O trabalho teve por objetivo determinar a influência da densidade de 10, 12, 14 e 16 aves $/ \mathrm{m}^{2}$ no desempenho de frangos de corte, durante a época de inverno. Aos 21 dias, já ocorreram diferenças de consumo, sendo o maior para as aves criadas na menor densidade (10 aves $/ \mathrm{m}^{2}$ ). O peso corporal foi influenciado pela densidade a partir dos 35 dias; o menor peso foi das aves com maior densidade, mas, não foi verificada influência sobre as características de carcaça avaliadas. A conversão alimentar não sofreu grandes variações, pois sempre que houve redução no peso corporal observou-se redução no consumo alimentar. A viabilidade criatória não foi influenciada pelas densidades avaliadas. Através de análise econômica concluiu-se que houve benefício econômico linear com o aumento da densidade de 10 para 16 aves $/ \mathrm{m}^{2}$.

Palavras-chave: frangos de corte, densidade de criação, inverno.

\section{SUMMARY}

The objective of this research was to determine the influence of the bird density: 10, 12, 14 and 16 birds/square meters on broilers performance, during winter time. After 21 days, the feed consumption was affected by different densities. The highest feed consumption was found on the lowest bird density (10 birds/square meters). The body weight was influenced by the density after 35 days. The lowest body weight was obtained on the highest density (16 birds/square meters). However, the carcass yield was not affected by density. The feed conversion was not affected significantil, When the body weight was reduced, the food intake was decreased too. The viability was not influencied by the densities. The economic analysis concluded that a linear benefit was found in increasing the bird density by 10 to 16 birds/square meters.

Key works: broilers, bird density, winter time.

\section{INTRODUÇÃO}

A cada dia os estudos nas áreas da genética e nutrição permitem uma rápida evolução no potencial produtivo das linhagens comerciais de frangos de corte e a adequação dos níveis nutricionais ao rápido desenvolvimento das aves, o que resulta em incremento no ganho de peso, conversão alimentar e rendimento de carcaça.

A crescente pressão para a redução dos custos de produção torna cada vez mais comum a prática de criação de frangos de corte em alta densidade, pois assim pode-se reduzir os custos com mão-de-obra e de investimentos na construção de novos aviários. Porém, na maioria das vezes, isso tem sido feito sem a necessária readequação de equipamentos, nutrição, manejo e ambiente em que as aves se encontram (LUCHESI, 1998).

ENGLERT (1974) não recomendava alojar mais de 10 aves $/ \mathrm{m}^{2}$, mas hoje existem trabalhos demonstrando que podem ser alojadas até

\footnotetext{
${ }^{1}$ Médico Veterinário, Professor do Colégio Agrícola de Santa Maria.

${ }^{2}$ Zootecnista, Professor, Departamento de Zootecnia, Universidade Federal de Santa Maria (UFSM), 97105-900, Santa Maria, RS. Email: Izanella@ ccr.ufsm.br. Autor para correspondência.

${ }^{3}$ Zootecnista.

${ }^{4}$ Médico Veterinário.

${ }^{5}$ Zootecnista, Mestre, Setor de Avicultura, UFSM.
} 
22 aves $/ \mathrm{m}^{2}$, o que resultaria num aumento significativo de produção de carne $/ \mathrm{m}^{2}$. PROUDFOOT et al. (1979), trabalhando com densidades de $10,13,18$ e 26 aves $/ \mathrm{m}^{2}$, concluíram que o aumento da densidade resultou em uma redução linear significativa de peso corporal em aves dos dois sexos e afetou a carcaça adversamente. BERENSKI (1987) obteve desempenho e características de carcaça semelhantes entre os tratamentos em experimento realizado com frangos de corte criados em lotações de 16, 18, 20 e 22 aves $/ \mathrm{m}^{2}$ até a oitava semana. MOUCHREK $\boldsymbol{e t}$ al. (1991) observaram que se pode criar até 14 aves $/ \mathrm{m}^{2}$ sem prejuízo fisiológico, principalmente em função da massa de carne produzida por área. CRAVENER et al. (1992) concluíram que é possível criar frangos de corte numa densidade que varia de 15 a 20 aves $/ \mathrm{m}^{2}$, obtendo resultados satisfatórios.

O objetivo deste trabalho foi avaliar a influência de diferentes densidades sobre o desempenho de frangos de corte criados na Região Sul do Brasil, no período de inverno, através da análise de índices produtivos.

\section{MATERIAL E MÉTODOS}

O experimento foi realizado no Setor de Avicultura do Departamento de Zootecnia da Universidade Federal de Santa Maria (UFSM), durante o período de inverno ( 2 de junho a 21 de julho de 1994), com temperatura média mínima de $10,8^{\circ} \mathrm{C}$, máxima de $20,6^{\circ} \mathrm{C}$ e média geral de $15,7^{\circ} \mathrm{C}$. Foram utilizados 1416 pintos de corte de um dia, da linhagem Cobb, os quais foram sexados através do empenamento da asa previamente ao alojamento, para a formação de lotes mistos compostos por $50 \%$ de machos e $50 \%$ de fêmeas, e pesados para determinação do peso médio inicial.

Utilizou-se o galpão experimental de frangos de corte do Setor de Avicultura do Departamento de Zootecnia da UFSM, composto por 52 boxes de $2,25 \mathrm{~m}^{2}$ de área $(1,5 \times 1,5 \mathrm{~m})$, dos quais foram utilizados 48 para o experimento. Os boxes foram previamente lavados e desinfetados e receberam como cama $10 \mathrm{~cm}$ de maravalha, como fonte de aquecimento uma campânula elétrica com lâmpada de 150 watts, um comedouro tipo bandeja e um bebedouro pendular. No $7^{\mathrm{o}}$ dia, os comedouros tipo bandeja foram substituídos por comedouros tubulares. Os equipamentos foram mantidos juntos por 24 horas para permitir a adaptação das aves. A campânula elétrica foi retirada no $15^{\circ}$ dia. Até o $5^{\circ}$ dia, as aves mortas ou descartadas foram substituídas.
As aves receberam ração inicial do $1^{\circ}$ dia de vida até o dia em que alcançaram consumo individual médio de $1,2 \mathrm{~kg} / \mathrm{ave}$. A ração crescimento foi oferecida até os 42 dias e a final dos 43 aos 49 dias. Durante todo o período de criação, as aves receberam alimentação ad libitum, com reposição sempre que necessário. Em todas as fases, utilizaram-se dietas a base de milho e farelo de soja e os níveis nutricionais utilizados foram $21,5,19,5$ e $17,5 \%$ de $\mathrm{PB}$ e 3050,3100 e $3150 \mathrm{kca} \ell \mathrm{EM} / \mathrm{kg}$ de dieta nas fases inicial, crescimento e terminação, respectivamente.

Foram avaliados quatro tratamentos, sendo $\mathrm{T} 1=10$ aves $/ \mathrm{m}^{2}, \mathrm{~T} 2=12$ aves $/ \mathrm{m}^{2}, \mathrm{~T} 3=14$ aves $/ \mathrm{m}^{2}$ e $\mathrm{T} 4=16$ aves $/ \mathrm{m}^{2}$. O delineamento experimental foi inteiramente casualisado. Foram avaliados os seguintes parâmetros: consumo alimentar, peso corporal, conversão alimentar, viabilidade criatória, características de carcaça e análise econômica. Os dados foram submetidos à análise de variância utilizando-se o programa SAEG e, quando as diferenças foram significativas ao nível de 5\%, aplicou-se o teste de Tukey.

Foram abatidas quatro aves ( 2 machos e 2 fêmeas) das 12 repetições por tratamento aos 35 dias, de 8 repetições aos 42 dias e de 4 repetições aos 49 dias, para a análise de carcaça. $\mathrm{O}$ abate foi realizado por sangria, a depena automática e evisceração manual. As carcaças foram pesadas com pés e cabeça para verificação de rendimento de carcaça e os cortes foram realizados manualmente e pesados para verificação de rendimento de perna e peito. A análise econômica deste trabalho foi baseada em um aviário para criação de frangos de corte padrão, com $1200 \mathrm{~m}^{2}$, sendo os resultados simulados com as 4 densidades avaliadas, utilizadose os resultados obtidos pelas aves nos diferentes tratamentos, aos 49 dias de idade. A análise foi feita apenas utilizando-se o custo dos pintos e da ração, que representam cerca de $90 \%$ dos custos de produção .

\section{RESULTADOS E DISCUSSÃO}

Na tabela 1, são apresentados o consumo de ração por ave e a conversão alimentar nas quatro densidades. Com base nos resultados, observou-se que a redução no consumo de ração é diretamente proporcional ao aumento da densidade dos 21 aos 49 dias, devido, principalmente, à restrição do acesso aos comedouros e bebedouros. Aos 21 dias, verificou-se diferença significativa de consumo $(\mathrm{P}<0,05)$ apenas entre o T4 $\left(16\right.$ aves $\left./ \mathrm{m}^{2}\right)$, que apresentou o menor consumo, e o T1 (10 aves $\left./ \mathrm{m}^{2}\right)$, que apresentou o maior consumo de ração. Dos 35 
Tabela 1 - Consumo (g) e Conversão alimentar em diferentes densidades e idades.

\begin{tabular}{lccccc}
\hline \multirow{2}{*}{$\begin{array}{c}\text { Densidade } \\
\text { (aves/m²) }\end{array}$} & \multicolumn{4}{c}{ Consumo alimentar $(\mathrm{g})$} & $\begin{array}{c}\text { Conversão } \\
\text { alimentar }\end{array}$ \\
\cline { 2 - 5 } & 21 dias & 35 dias & 42 dias & 49 dias & 49 dias \\
\hline T1- 10 & $997^{\mathrm{a}}$ & $2940^{\mathrm{a}}$ & $4240^{\mathrm{a}}$ & $5662^{\mathrm{a}}$ & 2,343 \\
T2- 12 & $970^{\mathrm{ab}}$ & $2836^{\mathrm{ab}}$ & $4180^{\mathrm{ab}}$ & $5444^{\mathrm{ab}}$ & 2,265 \\
T3- 14 & $925^{\mathrm{ab}}$ & $2793^{\mathrm{b}}$ & $4054^{\mathrm{b}}$ & $5231^{\mathrm{b}}$ & 2,252 \\
T4- 16 & $913^{\mathrm{b}}$ & $2540^{\mathrm{c}}$ & $3795^{\mathrm{c}}$ & $4995^{\mathrm{c}}$ & 2,218 \\
Média & 951 & 2777 & 4067 & 5333 & 2,280 \\
CV\% & 6,79 & 3,62 & 2,28 & 3,84 & 4,09 \\
\hline
\end{tabular}

Médias seguidas por letras desiguais nas colunas diferem entre si pelo teste de Tukey.

Tukey $(\mathrm{P}<0,05)$.

aos 49 dias, o consumo das aves submetidas ao T4 foi significativamente inferior ao do T3 $\left(14 \mathrm{aves} / \mathrm{m}^{2}\right)$ que, por sua vez, foi similar ao do T2 $\left(12\right.$ aves $\left./ \mathrm{m}^{2}\right)$ e inferior ao do T1. Resultados semelhantes foram encontrados por CAVALHEIRO et al. (1976), GRAÇAS et al. (1977), SULANE \& LEPAIYE (1978), BERENNSKI (1987) e LUCHESI (1998). Por sua vez, CONTE et al. (1997) não encontraram diferenças significativas no consumo alimentar de frangos criados em densidades de 10, 20, 30 e 40 aves $/ \mathrm{m}^{2}$ até os 21 dias, sendo que, após esta idade, todos os tratamentos possuíram 10 aves $/ \mathrm{m}^{2}$ até os 42 dias e consumos semelhantes. Os dados de conversão alimentar não apresentaram diferença significativa $\quad(\mathrm{P}>0,05), \quad$ confirmados por PARKUHURST et al. (1977), BRICENO et al. (1987) e LUCHESI (1998).

$\mathrm{Na}$ tabela 2, são apresentados os pesos alcançados pelas aves e a viabilidade criatória nos quatro tratamentos. Os resultados mostram que houve uma redução linear no peso corporal das aves com o aumento da densidade dos 21 aos 49 dias, embora, em altas densidades, os pesos tenham sido semelhantes aos obtidos pelas integrações avícolas do sul do Brasil. As baixas temperaturas ambientais seguramente contribuíram para o bom desempenho das aves em alta densidade, pois possibilitaram as trocas térmicas entre as aves e o ambiente, mantendo a temperatura corporal dos frangos na normalidade e gerando uma situação de conforto térmico. Até os 21 dias de idade das aves, não houve diferença significativa no peso corporal entre os tratamentos $(\mathrm{P}>0,05)$. Aos 35 dias, o peso corporal das aves do T4 $\left(16\right.$ aves $\left./ \mathrm{m}^{2}\right)$ foi significativamente inferior ao peso dos demais tratamentos, sendo que estes não diferiram entre si. Aos 42 e aos 49 dias, o peso
Tabela 2 - Peso corporal (g) e Viabilidade Criatória (VC) (\%) nas diferentes densidades e idades.

\begin{tabular}{|c|c|c|c|c|c|}
\hline \multirow{2}{*}{$\begin{array}{l}\text { Densidade } \\
\left(\text { aves } / \mathrm{m}^{2}\right)\end{array}$} & \multicolumn{4}{|c|}{ Peso corporal (g) } & \multirow{2}{*}{$\begin{array}{c}\text { VC } \\
49 \text { dias }\end{array}$} \\
\hline & 21 dias & 35 dias & 42 dias & 49 dias & \\
\hline $\mathrm{T} 1-10$ & 646 & $1588^{\mathrm{a}}$ & $2077^{\mathrm{a}}$ & $2416^{\mathrm{a}}$ & 97,73 \\
\hline $\mathrm{T} 2-12$ & 637 & $1544^{\mathrm{a}}$ & $2044^{\mathrm{a}}$ & $2405^{\mathrm{a}}$ & 98,15 \\
\hline T3- 14 & 628 & $1541^{\mathrm{a}}$ & $1983^{\mathrm{ab}}$ & $2323^{\mathrm{ab}}$ & 98,83 \\
\hline T4- 16 & 621 & $1480^{\mathrm{b}}$ & $1889^{\mathrm{b}}$ & $2234^{\mathrm{b}}$ & 98,65 \\
\hline Média & 633 & 1538 & 1998 & 2345 & 98,34 \\
\hline $\mathrm{CV} \%$ & 4,16 & 3,39 & 3,60 & 3,84 & 3,37 \\
\hline
\end{tabular}

Médias seguidas por letras desiguais nas colunas diferem entre si pelo teste de Tukey $(\mathrm{P}<0,05)$.

VC - Viabilidade criatória.

corporal dos frangos do tratamento $\mathrm{T} 4$ foi inferior aos pesos do T1 $\left(10\right.$ aves $\left./ \mathrm{m}^{2}\right)$ e T2 $\left(12\right.$ aves $\left./ \mathrm{m}^{2}\right)$ e semelhante ao T3 $\left(14\right.$ aves $\left./ \mathrm{m}^{2}\right)$. Pesquisadores como OLIVEIRA et al. (1969), GRAÇAS et al. (1977), SULANE \& LEPAIYE (1978), LEVINE \& FINK (1979), BERENSKI (1987), MAHAPATRA \& MOHAPATRA (1989), CRAVENER et al. (1992), SAKOMURA et al. (1997) e LUCHESI (1998) chegaram a resultados semelhantes.

A viabilidade criatória não foi influenciada significativamente pelas 4 densidades avaliadas $(\mathrm{P}>0,05)$. A não influência da densidade na viabilidade criatória foi confirmada pela maioria dos trabalhos revisados. (PARKUHURST et al. 1977, SULANE \& LEPAIYE, 1978, DELEV et al. 1986, DAFWAY et al. 1987 e BRICENO et al. 1987.)

Conforme a tabela 3 , aves criadas em diferentes lotações, quando abatidas até os 49 dias de idade, apresentaram rendimento de carcaça, perna e peito similares $(\mathrm{P}>0,05)$. Nas tabelas 4 e 5 , estão demonstrados os custos e as receitas nas diferentes densidades. Verificou-se que, ao elevar a lotação, aumenta-se a receita, o custo e o lucro do lote. O menor retorno econômico foi obtido com 10 aves $/ \mathrm{m}^{2}$, e o maior com 16 aves $/ \mathrm{m}^{2}$. Este resultado foi atribuído ao bom desempenho das aves criadas em altas densidades em relação ao seu consumo alimentar. O T1 (10 aves $\left./ \mathrm{m}^{2}\right)$ apresentou peso corporal $8,14 \%$ superior ao do $\mathrm{T} 4$, porém o consumo alimentar deste foi $11,78 \%$ inferior ao do T1. Estes resultados concordam com os resultados obtidos por PROUDFOOT et al. (1979) e LUCHESI (1998), que trabalharam com diferentes densidades concluíram que aumentando-se a lotação diminui-se a receita por ave, porém o lucro é maior quando calculado por área do galpão. 
Tabela 3 - Rendimento de carcaça $(\%)$, rendimento de perna (\% de coxa + sobrecoxa) e rendimento de peito (\%) nas diferentes densidades no período de inverno.

\begin{tabular}{|c|c|c|c|c|c|c|c|c|c|}
\hline \multirow{3}{*}{$\begin{array}{l}\text { Densidade } \\
.\left(\text { aves } / \mathrm{m}^{2}\right)\end{array}$} & \multicolumn{9}{|c|}{ Idade (dias) } \\
\hline & \multicolumn{3}{|c|}{35} & \multicolumn{3}{|c|}{42} & \multicolumn{3}{|c|}{49} \\
\hline & $\begin{array}{c}\text { Rendimento } \\
\text { carcaça }^{1}\end{array}$ & $\begin{array}{c}\text { Rendimento } \\
\text { perna }^{2}\end{array}$ & $\begin{array}{c}\text { Rendimento } \\
\text { peito }^{2}\end{array}$ & $\begin{array}{c}\text { Rendimento } \\
\text { carcaça }^{1}\end{array}$ & $\begin{array}{c}\text { Rendimento } \\
\text { perna }^{2}\end{array}$ & $\begin{array}{c}\text { Rendimento } \\
\text { peito }^{2}\end{array}$ & $\begin{array}{c}\text { Rendimento } \\
\text { carcaça }^{1}\end{array}$ & $\begin{array}{c}\text { Rendimento } \\
\text { perna }^{2}\end{array}$ & $\begin{array}{l}\text { Rend.imento } \\
\text { peito }^{2}\end{array}$ \\
\hline $\mathrm{T} 1-10$ & 77,97 & 26,49 & 24,28 & 77,43 & 27,87 & 25,33 & 79,53 & 27,43 & 25,17 \\
\hline $\mathrm{T} 2-12$ & 78,04 & 27,14 & 24,14 & 78,68 & 28,62 & 24,66 & 78,31 & 27,26 & 26,26 \\
\hline T3- 14 & 78,37 & 25,97 & 23,48 & 78,00 & 27,96 & 24,44 & 78,41 & 27,21 & 26,06 \\
\hline T4- 16 & 79,42 & 27,43 & 24,19 & 78,92 & 27,91 & 25,05 & 80,75 & 27,44 & 25,37 \\
\hline Média & 78,46 & 26,81 & 24,06 & 78,26 & 28,09 & 24,87 & 79,25 & 27,34 & 25,72 \\
\hline $\mathrm{CV} \%$ & 1,67 & 3,70 & 3,81 & 1,96 & 2,39 & 4,70 & 2,03 & 1,63 & 2,29 \\
\hline
\end{tabular}

${ }^{1}$ Carcaça com pés e cabeça e porcentagem em relação ao peso vivo.

${ }^{2}$ Porcentagem em relação à carcaça.

Tabela 4 - Número inicial de aves, consumo total de ração $(\mathrm{kg})$, custo total da ração/lote (R\$), número final de aves, custo de pintos (R\$), peso médio do lote $(\mathrm{g})$ e peso total do lote $(\mathrm{kg})$.

\begin{tabular}{lccccccc}
\hline Dens. aves $/ \mathrm{m}^{2}$ & $\mathrm{~N}^{\circ}$ inicial de aves & Consumo total & Custo ração & $\mathrm{N}^{\mathrm{o}}$ final aves & Custo pintos & Peso médio & Peso total lote \\
\hline T1- 10 & 12.000 & 67.944 & 14.268 & 11.728 & 4.080 & 2416 \\
T2-12 & 14.400 & 78.394 & 16.463 & 14.134 & 4.896 & 28.335 \\
T3- 14 & 16.800 & 87.881 & 18.455 & 16.603 & 5.712 & 2323 \\
T4- 16 & 19.200 & 95.904 & 20.140 & 18.941 & 6.528 & 2234 \\
\hline
\end{tabular}

Preço kg ração - R \$ 0,21

Preço pinto de um dia (unidade) - R \$ 0,340.

FONTE: Setor de Avicultura da Universidade Federal de Santa Maria.

Tabela 5 - Receita/lote (R\$), custo/lote (R\$), lucro (R\$), custo/kg vivo $(\mathrm{R} \$)$ e custo/área $\left(\mathrm{R} \$ / \mathrm{m}^{2}\right)$.

\begin{tabular}{lccccc}
\hline $\begin{array}{l}\text { Densidade } \\
\text { (aves/m }\end{array}$ & Receita & $\begin{array}{c}\text { Custo/ } \\
\text { lote }\end{array}$ & Lucro/lote & $\begin{array}{c}\text { Custo/ } \\
\mathrm{kg}\end{array}$ & $\begin{array}{c}\text { Custo/ } \\
\mathrm{m}^{2}\end{array}$ \\
\hline T1-10 & 21.251 & 18.348 & 2.903 & 0,65 & 15,29 \\
T2-12 & 25.494 & 21.359 & 4.135 & 0,63 & 17,80 \\
T3-14 & 28.927 & 24.167 & 4.760 & 0,63 & 20,14 \\
T4- 16 & 31.736 & 26.668 & 5.068 & 0,63 & 22,22 \\
\hline
\end{tabular}

Preço kg de frango - $\mathrm{R} \$ 0,750$

FONTE: Setor de Avicultura da Universidade Federal de Santa Maria.

\section{CONCLUSÃO}

O consumo alimentar e o peso corporal são superiores nas aves criadas em menores densidades, porém conversão alimentar, viabilidade criatória e as características de carcaça não são afetadas pela densidade de criação. Pode-se concluir que, na Região Sul do Brasil, no inverno, pode-se aumentar a densidade de 10 para 16 aves $/ \mathrm{m}^{2}$, com maior retorno econômico por lote de frangos.

\section{REFERÊNCIAS BIBLIOGRÁFICAS}

BERENSKI, C.H. Rearing broilers at different stocking density and lengths of fattening. Basic produtives characteristics. Zhivotnov dni-nauki, Bulgária, v.24, n.1, p.20-25, 1987.

BRICENO, W.N.O., GUIMARÃES, F.C.R., CRUZ, F.G.G. Efeitos da densidade populacional de frangos de corte em época quente. Município de Manaus. In: CONGRESSO BRASILEIRO DE AVICULTURA, 1987, Campinas, SP. Anais... Campinas : FACTA, 1987. p.131-132.

CAVALHEIRO, A.C.L., OLIVEIRA, S.C., TRINDADE, D.S., $\boldsymbol{e} t$ al. Determinação do espaço de acesso por ave nos comedouros tubulares na criação de frangos de corte. Anuário Técnico IPZFO, Porto Alegre. v.1, n.4, p.158-162, 1976.

CONTE, A.J., COTTA, J.T.B., TEIXEIRA, A.S., et al. Efeitos das densidades iniciais de criação e da transferência de alojamento aos 21 dias de idade no desempenho de frangos de corte. In: CONFERÊNCIA APINCO, 1997, São Paulo-SP. Trabalhos de Pesquisa... Campinas : FACTA, 1997. p.4.

CRAVENER, T.L., ROUSCH, W.B., MARSHALY, M.M. Broiler production under varying population densities. Poultry Science, v.71, n.3, p.427-433, 1992. 
DAFWANG, I.I., COOK, M.E., SUNDE, M.L. Interaction of antibiotic supplementation and stocking density on broiler chick performance and inmune response. British PoultryScience, Inglaterra, v.28, n.1, p.47-55, 1987.

DELEV, K., DZKANOV, T., JANKOV, T. Influence of the cage density of broilers on morbidity and mortality. Veternarnomeditsinski-Nauki, Bulgária, v.23, n.1, p.67-72, 1986.

ENGLERT, S. Produção de frangos para corte. Avicultura. Porto Alegre : Centaurus, 1974. p.126-191.

GRAÇAS, A.S., FONSECA, J.B., SOARES, P.R., et al Determinação do espaço de piso para criação de frangos de corte. In: REUNIÃO ANUAL DA SOCIEDADE BRASILEIRA DE ZOOTECNIA, 1977, Recife-PE. Anais..., Campinas : FACTA, 1977. p. 141.

LEVINA, L.I., FINK, L. Production traits of broilers in relation to stocking densyty during rearing. Trudy-Alma-AtinskogoZoo-Veterinarnogo-Institute-Russian, v.34, p.14-17, 1979.

LUCHESI, J.B. Custo-benefício da criação de frangos de corte em alta densidade no inverno $\mathrm{e}$ no verão. In CONFERÊNCIA APINCO, 1998, Campinas-SP. Anais..., Campinas : FACTA, 1998. p.241-248.

MAHAPATRA, C. M., MOHAPATRA, S. C. Effect of stock density on meat yield and quality of broilers. Indian Journal of Animal Science, v.59, n.7, p.903, 1989.
MOUCHREK, E., TANAKA, T., LINHARES, F., $\boldsymbol{e} \boldsymbol{t} \boldsymbol{a}$. Crescimento de frangos de corte criados em alta densidade populacional na época fria. In: CONGRESSO BRASILEIRO DE AVICULTURA, 1991, Santos-SP. Anais... Campinas : FACTA, 1991.p.123.

OLIVEIRA, S.C., TRINDADE, D.S., PAOLI AZEVEDO, L.A., et al. Influência da adição de gorduras às rações e da lotação na criação de frangos de corte. Porto Alegre : Departamento de Produção Animal, Secretaria da Agricultura, 1969. 9p. (Boletim Técnico).

PARKUHURST, C.R., BAUGHMAN, G.R., THAXTON, J.P., et al. A comparison of broilers grow in environmentaly modified an conventional housing at different population densities. Poultry Science, v.56, p.883-885, 1977.

PROUDFOOT, F.G., HULAN, H.W., RAMEY, D.R. The effect of four stocking densities on broilers carcass grade, the incidence of breast blisters, and other peformance traits. Poultry Science, v.58, n.4, p.791-793, 1979.

SAKOMURA, N.K., OLIVEIRA, J.E., FIGUEIREDO, A.N., et al. Efeito do isolamento térmico sobre o desempenho de frangos de corte alojados em diferentes densidades. In: CONFERÊNCIA APINCO, 1997, São Paulo-SP. Trabalhos de Pesquisa... Campinas : FACTA, 1997. p.3.

SULANE, E., LEPAIYE, L. High stocking density of broilers. Ptitsevodstvo, Russian, n.5, p.25-27, 1978. 